\title{
La sensorialidad como estrategia para la educación patrimonial en el aula de educación infantil
}

\author{
Sensuousness as equity strategy for education in the classroom of \\ childhood education
}

\author{
Carmen GÓMEZ. Universidad de Valladolid (España). \\ carmen.gomez.redondo@gmail.com \\ Beatriz FENOY. Universidad de Valladolid (España). \\ trizabe.fm@gmail.com
}

Resumen: Con este artículo se pretende poner en valor la actividad sensorial como estrategia para la educación patrimonial en el aula de infantil. Tras una primera parte de revisión teórica, el artículo pretende demostrar, a través de un diseño educativo realizado al efecto e implementado en el aula de infantil, la relación entre la sensorialidad y los procesos de patrimonialización en edades tempranas. Este diseño nos permite establecer una secuencia didáctica que parte del conocimiento sensorial, como base del aprendizaje significativo en la etapa de infantil y que permite establecer canales para la vinculación afectiva entre sujeto y objeto.

Palabras clave: Educación patrimonial, actividad sensorial, procesos de patrimonialización, educación infantil.

\begin{abstract}
This article aims to give value to the sensory activity as a strategy for heritage education in the classroom child. After a first part of theoretical review, the article seeks to demonstrate, through an educational purpose design made and implemented in the classroom child, the relationship between the sensory and heritagization processes at an early age. This design allows us to establish a teaching sequence that part of sensory knowledge, as a basis for meaning full earning in the infant stage and allows to establish channels for the emotional connection between subject and object.
\end{abstract}


Keywords: heritage education, sensory activity, heritagization processes, early childhood education.

\section{Introducción}

Tal vez no resulte necesario traer aquí, de nuevo, los paradigmas surgidos en torno a la concepción de patrimonio, ni definir el término más allá de los monumentos y elementos artísticos. Como ya ha defendido Fontal en diversas publicaciones: "El patrimonio es la relación entre bienes y personas" (Fontal, 2014, p.18) y que han sido defendidos y repensados por otros autores referentes Huerta y de la Calle (2013), Calaf (2009), Juanola, Calbó y Vallés (2006), Cuenca (2014), Vicent e Ibáñez (2012). Así pues, partimos de este planteamiento paradigmático en el que el patrimonio deja de ser un acervo de elementos para ser la forma en que las personas se relacionan con esos elementos.

Dicha perspectiva más humanista del patrimonio evidencia el protagonismo de la acción educativa para mediar en estas relaciones. Sin embargo, en la puesta en práctica de estos principios se plantea el primer requisito que, a modo de eslabón, marcará el resto del proceso, la adecuación de los diseños y propuestas educativas al perfil del público o alumnado y al contexto.

No es difícil imaginar el crisol de oportunidades que brinda el conjugar las diferentes variables que intervienen, al diseñar este tipo de propuestas atendiendo a los agentes y su contexto. En este artículo se pretende mostrar una de esas posibles conjugaciones a través de un diseño didáctico que parte de la sensorialidad para acertar el patrimonio al público infantil. Nuestra propuesta, además viene a ampliar el acervo de diseños que, desde la educación patrimonial, se vienen proponiendo para ámbitos educativos formales. Este engrose, además es una puesta en valor de la educación patrimonial en este entorno y una llamada a la necesidad de su empleo desde edades tempranas.

A lo largo de este artículo, a través de una revisión teórica, se justifica por qué la idoneidad de lo sensorial para acercar el entorno a los niños de esta etapa, así como la necesidad de la intervención desde la educación patrimonial en este acercamiento. Esta necesidad es la que nos ha llevado a plantear el diseño de intervención que se resume a continuación, para finalmente proponer una secuencia procedimental para la detonación de procesos de patrimonialización en el aula de infantil.

\section{La sensorialidad como estrategia en la educación infantil}

Los sentidos son puertas abiertas al mundo y a la realidad, es decir, es el canal de comunicación entre el individuo y su entorno, el mundo que construimos es por tanto, el resultado de esta percepción. Esta construcción del mundo subjetiva y 
dependiente de la percepción ponen de manifiesto la necesidad de estimulación y educación en edades tempranas. González (2011) asegura que:

En los primeros años de vida, los sentidos son el medio que permite a los niños y niñas conocer el mundo que les rodea. A medida que van desarrollando sus sentidos, van progresando en el conocimiento y la construcción de un mundo físico y social cada vez más completo. De ahí, la importancia de trabajar en el aula de educación infantil la estimulación y desarrollo sensorial. (citado en Cardo y Vila, 2005, p. 6)

Sin embargo, aunque los sentidos son la puerta de entrada del entorno en el individuo, esta información no pasa directamente hacia el ámbito de la cognición y el aprendizaje, sino que primeramente generan una respuesta automática (más adelante sostendremos que esta respuesta se produce desde la afección) que marcará el tipo de aprendizaje y el manejo que se haga a nivel cognitivo con esa información, sentido y sensación, por tanto, van estrechamente unidos. Teniendo en cuenta que la sensación producida por el sentido marca el tipo de aprendizaje y los procesos cognitivos que rigen la gestión de esa información, queda evidenciada la necesidad de intervención educativa en esas primeras percepciones, procurando que esas primeras experiencias sensitivas sean conscientes. Soler (1992), afirma que:

La vida de relación con el mundo que rodea al niño se inicia a través de los sentidos y por las sensaciones comienza su mente a construir sus propias ideas; por eso, desde el principio, es necesaria la guía en la interpretación de las impresiones sensitivas, en la aclaración de las percepciones sensibles. (citado en Cardo y Vila, 2005, p. 30)

Así pues, la educación sensorial plantea, como cualquier contenido programado en un aula, una serie de objetivos que permiten una intervención educativa. Soler (1992), plantea algunos de los objetivos docentes que deben formularse para una educación sensorial, centrándose en la consciencia y organización de la percepción a través de la estimulación y el fomento de las experiencias sensoriales:

- Fomentar, potenciar y mejorar la capacidad sensorial.

- Estimular el desarrollo cognitivo mediante la educación sensorial.

- Ser capaz de estructurar la información que se obtiene a través de los sentidos.

- Fomentar la adquisición del conocimiento mediante la programación y desarrollo de actividades multisensoriales que permitan la manipulación y la experimentación ya sea mediante objetos durante un juego o aprovechando los recursos que ofrece el entorno. (Citado en Cardo y Vila, 2005, p. 52) 
En este caso se plantea una de las claves para el empleo de la sensorialidad como estrategia de aprendizaje: la relación entre la sensorialidad y el desarrollo cognitivo, la estimulación sensorial lleva directamente al aprendizaje, pero un aprendizaje más complejo que implica lo cognitivo y lo físico. Esto nos lleva a deducir, por obvio que parezca el resultado, que a través de la sensorialidad y la experimentación se llega al conocimiento del entorno. Así pues, no solo conocer la composición de un mineral es conocimiento sobre ese mineral, también lo será saber a qué huele, cómo sabe, si es pesado o frío y qué colores y texturas tiene. Llamar la atención sobre esta obviedad no viene sino a poner de relieve la importancia de la experimentación para llegar al aprendizaje significativo, sobre todo, en estos primeros años.

Finalmente la sensorialidad como forma de conocimiento y -como defendemos en el punto siguiente- la afección como materia consolidante de este conocimiento, son las bases para la posterior propuesta didáctica, que supone una aproximación de la educación patrimonial en la educación infantil y un cuerpo empírico que sustente un itinerario procedimental para la patrimonialización en las primeras etapas educativas.

\section{De la percepción a la emoción y de la emoción al afecto}

A la luz de lo expuesto anteriormente parece que el gran canal de entrada hacia la cognición son los sentidos. Sin embargo ¿Qué pasa una vez que el entorno ha entrado en el terrero de lo subjetivo? Para responder a esta pregunta nos serviremos de la propuesta planteada por Damasio (1999), apartándonos de su perspectiva neurofisiológica, tomamos la relación entre percepción/emoción/sentimiento como un proceso. De esta manera, del primer contacto sensorial del individuo con el objeto aparece una respuesta fisiológica, una emoción, asociada a ciertas manifestaciones del cuerpo como pulsación, sudoración, etcétera. Percepción y emoción son construidos como sentimientos según Damasio (2000) que finalmente se asocian al recuerdo, o imagen mental de dicho objeto. Así pues, podríamos decir que el aprendizaje a través de la percepción sensorial implica la detonación de emoción que se transforma en sentimiento.

Percepción y sentimiento se relacionan y ordenan dentro del individuo como lo aprendido, de manera que una nueva percepción del elemento detonante derivará de nuevo en el sentimiento asociado, pero también ocurrirá a la inversa, la recreación del sentimiento llevará al recuerdo del elemento:

Esto significa que todo lo que edifique la fantasía influye recíprocamente en nuestros sentimientos, y aunque ese edificio no concuerde, de por sí, con la realidad, todos los sentimientos que provoque son reales, efectivamente vividos por el hombre que los experimenta (Vigotsky, 1998, p. 23).

Además, Vigotsky añade que las distintas percepciones pueden relacionarse en base a una misma emoción, las percepciones emocionalmente cargadas en un 
sentido tienden a asociarse en ciertas categorías afectivas, tomando en parte una esencia común, creando por tanto redes de recuerdos en base a emociones comunes, detonadas por percepciones diferentes en base a distintos elementos del entorno.

Dentro de la gama de sentimientos que puede construir un individuo ante una percepción y emoción, comprendemos que estos se producen conjugados por una potencia más básica aún a la que denominamos filia:

En primer lugar daremos cuenta de esta potencia tan básica, tan primaria al ser humano que apenas es definible tal vez como inclinación en su percepción más básica. Se hace necesario recurrir a la semántica y al lenguaje para establecer un cosmos referencial que de cuenta además, de su posible gradación y polaridad. Partiendo de una primera percepción podríamos establecer esta relación afectiva en términos de: percepción de existencia, curiosidad, motivación, inclinación, propensión, preferencia, importancia, afinidad, simpatía, atracción, afición, proximidad, consideración, estima, aprecio, apego, valoración, devoción, cariño, afecto, ternura, amor, pasión, adoración, idolatría. En su cualidad polar negativa podríamos establecer una gradación similar: percepción de existencia, desinterés, retroceso, rehusar, antipatía, rechazo, animadversión, repeler, repudiar, antipatía, aversión, odio. Esta nube de términos como acciones da cuenta de las gradaciones que cualitativamente acerca y aleja a los sujetos a través de lo no cognitivo.

Esta filia a la que referimos interacciona con la gama de sentimientos que se han dibujado tradicionalmente desde la psicología y ahora desde la neurociencia: alegría $^{1}$, deseo, tristeza, admiración, desprecio, propensión, aversión, devoción, esperanza, miedo, seguridad, desesperación, gozo, remordimiento, conmiseración, aprobación, indignación, sobreestimación, menosprecio, etcétera. Actuando de plusvalía, incrementando la gradación del sentimiento. De esta forma, el sentimiento de alegría se puede ver incrementado si la alegría ha sido provocada por un objeto sobre el que se ha establecido una relación en base a esta filia. Del mismo modo ocurrirá con el resto de sentimientos, viéndose contaminados de esta filia, que añade valores a la gradación propia del sentimiento. (Gómez-Redondo, 2014, p. 68-69)

Finalmente, de lo descrito hasta aquí parece querer dibujarse un camino hacia la patrimonialización -es decir, a la apropiación simbólica del entorno- a través de la percepción sensorial, que como se ha defendido anteriormente, es el canal básico de aprendizaje en las primeras etapas de desarrollo.

1. Se enuncian en este listado algunos de los sentimientos propuestos por Spinoza (1958). 


\section{Diseño de una propuesta de educación patrimonial a través de la percepción sensorial}

Una vez expuestas las razones que justifican la introducción de la educación patrimonial como contenido educativo a través de la sensorialidad exponemos el diseño de una propuesta educativa ajustada a las necesidades, objetivos y contenidos de la etapa infantil. Esta propuesta nacecon el fin de generar emociones que den relevancia al proceso y a la forma en que las personas se relacionan con los elementos creando un vínculo sólido. Pese a que, en este caso concreto, el diseño de educación patrimonial se plantee en forma de unidad didáctica, la complejidad y las numerosas posibilidades que abarca la temática permitirían trabajar este contenido en las aulas a través de otras metodologías.

Esta propuesta educativa se plantea desde un principio introductorio, con el fin de iniciar al alumnado de infantil en la temática a través de una primera experiencia de patrimonialización del entorno próximo (el entorno próximo al centro, contextualizado en el barrio de Nueva Segovia (Segovia)). Este carácter iniciático quedará reflejado en el planteamiento de los objetivos y contenidos en base a la propuesta de taxonomías de Bloom (1972), empleando términos relacionados con las fases iniciales del aprendizaje y desde un enfoque más patrimonial se tendrá en cuenta la secuencia procedimental de Fontal (2003).

Siguiendo los principios de la educación infantil en el ámbito formal, el planteamiento del diseño parte de la intención de generar una motivación e interés en el alumnado que conecte con su experiencia previa Calaf y Fontal (2007), para ello se diseñan dos actividades iniciales que tienen como finalidad recopilar centros de interés para mantener despierta la motivación de los niños y que la propuesta pueda ser realmente significativa para ellos. Dichas actividades ayudan al docente a descubrir qué zonas del entorno próximo son de su interés (potenciales elementos patrimoniales) y le permiten generar, en conjunto, una ruta que recoja las zonas escogidas en las que, posteriormente, se realizarán las actividades sensoriales que generen ese vínculo y conexión entre patrimonio, emoción y expresión de sensaciones.

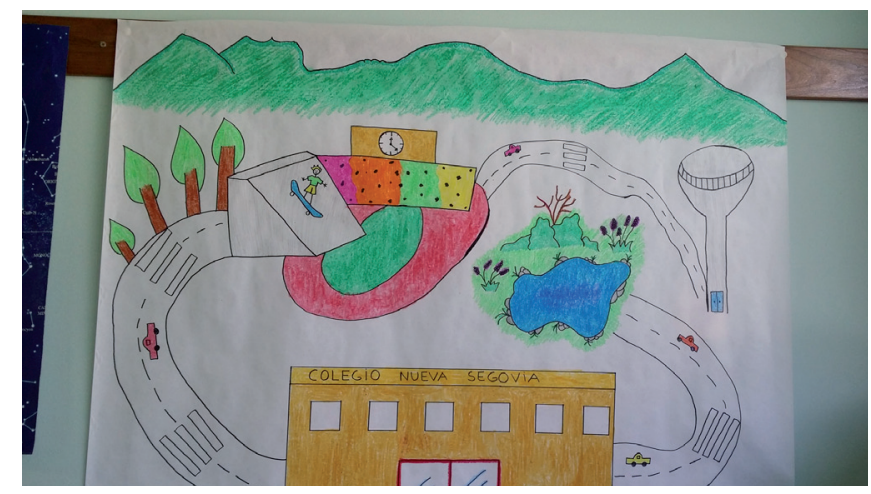

Figura.1. Ejemplo del diseño de la ruta establecida por el alumnado en forma de mapa. 
Tras esta primera parte de detección de intereses, la unidad didáctica se estructura en cuatro ejes principales: las texturas, los colores, los olores y la creación final de un Lapbook ${ }^{2}$ como actividad de recopilación y evaluación final. Los tres primeros ejes presentan el mismo guión de trabajo con diferentes actividades aplicadas, centrándose en los distintos sentidos:

1․ Secuencia de actividades introductorias: a través de una asamblea inicial y de una pequeña actividad se presenta el sentido con el que vamos a trabajar.

$2^{\circ}$. Secuencia de actividades de desarrollo: consiste en la salida al entorno siguiendo la ruta establecida por los alumnos y en la que se realizarán las actividades sensoriales diseñadas a tal efecto. Estas serán las actividades más relevantes y las que determinarán el éxito o no de la propuesta. Los niños interaccionan con su entorno próximo y se inician en la creación de un vínculo entre la percepción sensorial del elemento y la emoción detonada.

$\underline{3^{\circ} .}$ Secuencia de actividades finales: estas actividades tienen la intención de trabajar con todas las emociones y experimentaciones que han vivido a través de la interacción sensorial con los elementos. Este trabajo de integración, tiene como fin de generar una estructura patrimonial, un cosmos de conocimiento y patrimonialización. La expresión plástica tiene aquí un protagonismo esencial, es canal de comunicación, pero además tiene un carácter performático, los niños construyen su patrimonio y a sí mismos a través de la expresión plástica.

Ejemplifiquemos pues, a través de imágenes y una breve descripción, algunas de las actividades principales de la propuesta, una por cada sentido trabajado en el aula:

- Las texturas: en el parque del barrio los niños perciben colores, olores, formas... hasta que se les plantea la posibilidad de tocar y reflexionar sobre ello. Una vez que descubren que existen numerosas texturas en una misma zona, plasman en un folio a través de la técnica del frotage las texturas que más llamen su atención: el tronco rugoso de un árbol, la madera quebrada de los bancos, las formas de las hojas, etc.

- Los olores: en uno de los entornos escogidos los participantes conocen a través del olfato. En esta actividad se parte de una serie de preguntas y planteamientos que les sirvan de guía: ¿huelen todas las flores iguales? ¿tiene olor una piedra? ¿qué flores están más presentes? De este modo el niño descubre qué olores son los más presentes en su entorno así como la flora característica de la zona geográfica.

2. Se emplea el término Lapbook para denominar un nuevo soporte de trabajo visual, a caballo entre el poster y el cuaderno, en el que se plasman los principales conceptos de un tema de interés trabajado. 


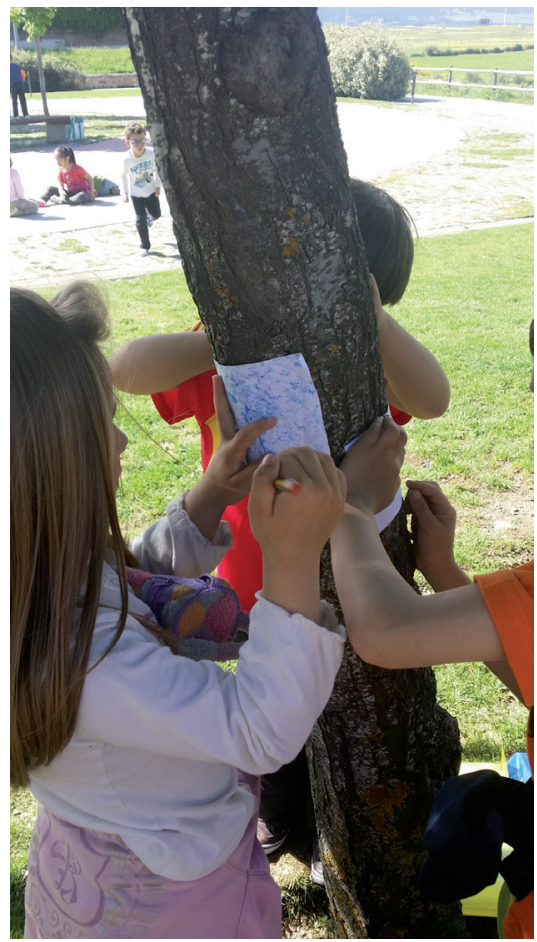

Figura 2. Ejemplo de actividad sensorial dentro de su entorno próximo en relación a las texturas.

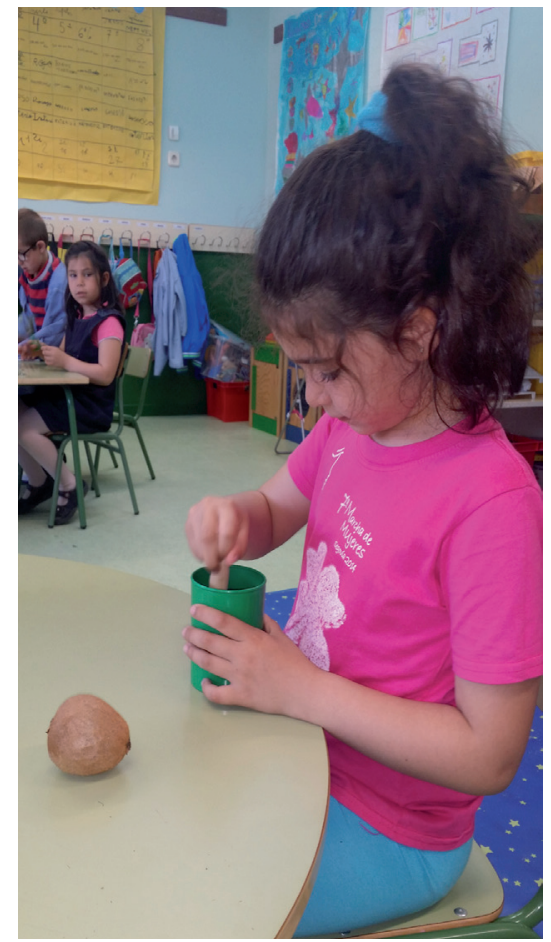

Figura 3. Ejemplo de actividad sensorial en el aula a través de la previa interacción con el entorno a partir del olfato.

Con el material recogido y las emociones y experiencias que ha generado el olor, procedemos a crear entre todos "El perfume Segoviano" compuesto por dos plantas típicas de la zona: romero y cantueso y, por una fruta que seleccionada por los niños: el kiwi. Los niños machacan las flores y el kiwi para extraer la esencia mientras huelen sus propias manos y recuerdan el lugar en el que recogieron las flores.

- Los colores: en el patio del colegio, con tizas de colores, dibujan cualquier aspecto, elemento o detalle que haya sido de su interés en la interacción con el entorno, haciendo memoria de los colores que estos tenían. Algunos son fieles a la realidad y otros prefieren dibujarlos tal y como les gustaría que fuesen. El color del entorno entra en el centro, se integra en el entorno. El espacio escolar se integra en este proceso de puesta en valor y patrimonialización del entorno. 


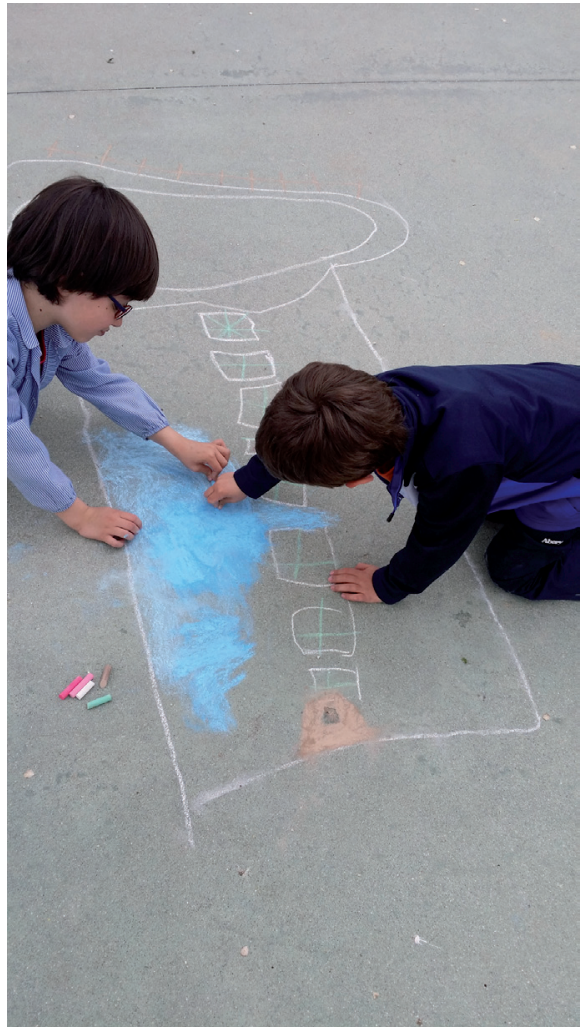

Figura.4. Ejemplo de actividad sensorial en las instalaciones del colegio a través de la previa interacción con el entorno mediante los colores percibidos.

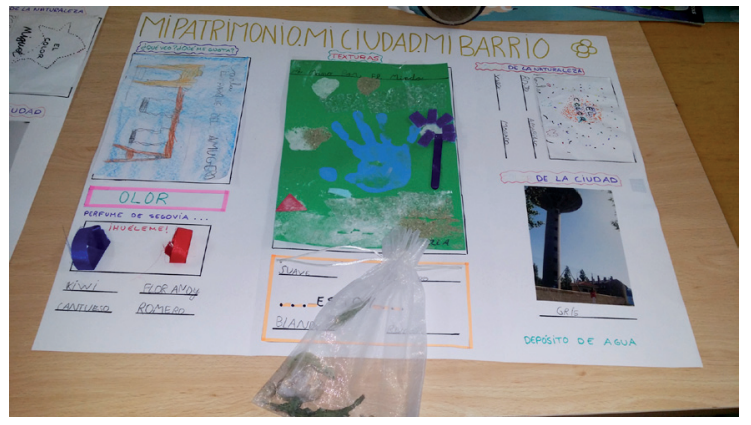

Figura 5. Ejemplo de actividad final que recopila los contenidos y resultados más relevantes de la propuesta.

Por último, diseñamos una actividad final que engloba todo lo que se ha trabajado a lo largo de la propuesta y que sirve, no solo de evaluación, sino de recordatorio para ellos mismos y para la comprobación del éxito de la propuesta a través de sus gestos, expresiones, respuestas, etc. En este caso, se optó por el diseño de un Lapbook que recoge algunos de los aspectos más relevantes del entorno a través de cada uno de los sentidos trabajados dentro y fuera del aula. Este trabajo es también una síntesis del proceso de patrimonialización, podríamos decir que incluso se convierte en seña de identidad del grupo y por tanto en su patrimonio del presente Fontal (2006), creado y no heredado.

\section{Aportaciones de la dimensión sensorail a la educación patrimonial en educación infantil}

El patrimonio es todo aquello que un individuo valora, aquello que forma parte de su entorno, de su vida y su experiencia. Todo valor o sentimiento (ya sea de rechazo o de aceptación) parte de una sensación previa, de una primera toma de contacto y de todas las experiencias que se hayan generado en relación a él. Involucrar la experimentación y cada uno de los sentidos permite generar todas estas 
observaciones y hacer que el individuo se tome un papel agente en la valoración de aquello que es o no relevante en su entorno y por qué.

Planteada esta reflexión en el contexto escolar Cuenca (2014), surge la necesidad de preguntarse: ¿cuál es el camino para generar la patrimonialización que estamos defendiendo dentro de las aulas?, ¿podemos hablar de metodologías o estrategias concretas que consoliden el vínculo patrimonial entre individuo-elemento adaptadas a las necesidades evolutivas del individuo? Es aquí donde surge la experimentación sensorial como respuesta a éstas preguntas. La experimentación en sí implica la participación activa y directa del alumnado de educación infantil, lo que fomenta un aprendizaje significativo en el que el alumno construye su propio conocimiento combinando conocimientos previos y conocimientos nuevos. Si el alumno es el propio generador de ideas, el proceso de enseñanza-aprendizaje se vuelve sólido y el conocimiento se asienta y asimila de manera más eficaz.

La experimentación como forma de aprendizaje no sería posible sin la intervención directa de los sentidos: tocar, oler, probar, escuchar y ver todo lo que el entorno puede ofrecer, pero es necesario añadir que en ese aprendizaje aparece también la emoción como forma de generar sentimientos: rechazo, admiración, satisfacción, pertenencia, aprobación, disfrute, frustración, etc. De éste modo, el alumno va ordenando sus experiencias/aprendizajes en función de las sensaciones y los sentimientos por ellas generados. Aprender patrimonialmente, significa precisamente esto, generar aprendizajes sentidos, de tal forma que lo aprendido conforme parte del patrimonio y la identidad del individuo, el aprendizaje sentido supone apropiación del elemento, pertenencia e identidad como expresa Fontal (2003).

La experiencia propuesta viene a confirmar que la expresión artística se configura, realmente, como un catalizador del proceso de patrimonialización. La experimentación y la emoción se digieren y catalizan a través de la expresión artística, aprovechando la capacidad de este medio para permear y profundizar en el interior del individuo, tal y como defiende Huerta (2013). Esta expresión permite al niño poner en orden sus emociones y sensaciones, a través de ella es capaz de ordenarlos según un valor determinado. Todo ese proceso, desde la idea principal hasta el resultado final, determinará el valor final de lo que él irá considerando como su propio. De ese modo, el patrimonio se convierte en lo que realmente es: un arrecife común, construido a partir de relatos individuales según Gómez-Redondo (2013).

Esta experiencia además, nos ha permitido distinguir puntos de interés, elementos clave que aparecen a lo largo de la investigación. Estos elementos se tornan a nuestros ojos como estrellas de una constelación que permite dibujar un posible proceso de patrimonialización en la etapa de educación infantil. Esta constelación puede traducirse en una secuencia didáctica, una estructura de finalidades que orienten el proceso de patrimonialización en la etapa de infantil. Esta constelación la podemos concretar en la secuencia: percepción- emoción- expresión- patrimonialización, que queda pormenorizada a continuación. 


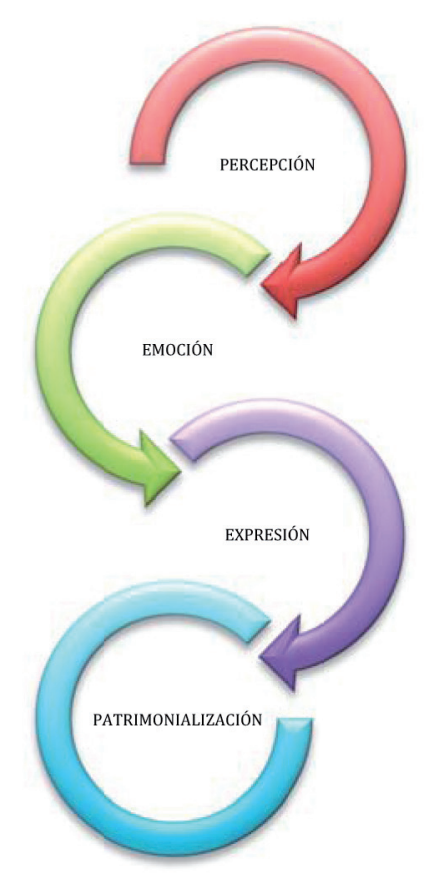

Figura 6. Secuencia didáctica para la patrimonialización desde la experimentación.

- Percepción: no podemos hablar de percepción sin sensación. Percibir es la acción de asimilar un conocimiento a través de las impresiones que generan los diferentes sentidos frente a un objeto, situación, etc. No se considera o se considerará patrimonio aquello que no genere una impresión o emoción ya que no llegará a consolidarse como vínculo entre objeto y sujeto.

- Emoción: si hablamos de educación patrimonial como proceso, entendemos que una cosa lleva a la otra. La oportunidad de percibir genera en el sujeto la capacidad de emocionarse. El rechazo o la aprobación, la fascinación o desagrado, la risa, el llanto, una mueca, determinan la consideración personal de patrimonio. Las emociones permiten al sujeto no sólo formar parte de un vínculo sino sentirse parte de él. Las emociones serán la clave del patrimonio como forma de vida individual y conjunta y generará en el individuo la necesidad de cuidarlo, mantenerlo y compartirlo.

- Expresión/ construcción del sentimiento: ¿podríamos entender las emociones si sólo fueran definiciones?, ¿podríamos entender la tristeza sin lágrimas, sin el rostro fruncido? ¿y la alegría sin una sonrisa? Es más, ¿entenderíamos la tristeza sin tener la necesidad de escuchar música melancólica?, ¿y la alegría sin canto? Una emoción existe cuando se expresa. Son numerosas las formas de manifestar una emoción: el arte y la plasticidad, son una buena opción para la educación patrimonial ya que en ocasiones precisamos que esa expresión 
sea mantenido en el tiempo, o bien para nosotros mismos o para compartirlo con el mundo. Por lo tanto podríamos afirmar para este trabajo que no hay emoción sin percepción y no hay expresión sin emoción. Si logramos formar y poner en marcha este primer engranaje, estaremos hablando sin duda de un germen de la patrimonialización.

Una emoción es una respuesta involuntaria que surge a través de las impresiones de un objeto o situación. El sentimiento va más allá, es ser consciente no sólo de la existencia de una emoción sino de sentirla, mantenerla en el tiempo y asociarla al elemento. El ser humano es capaz de sonreír sin ser feliz, de llorar sin sentir tristeza, de gritar sin sentir rabia y justo ahí está la diferencia entre emoción y construcción del sentimiento. Toda percepción genera una emoción pero no todas las percepciones generan sentimiento. Sólo aquellas experiencias que se sienten interna y conscientemente formarán parte del desarrollo del individuo y definirán las bases sólidas del vínculo entre objeto-sujeto, dando forma a la valoración personal de patrimonio. Ser consciente de sentir una emoción no es tarea sencilla $y$, en numerosas ocasiones, la incapacidad por controlar o reconocer un sentimiento distorsiona la realidad y repercute en el desarrollo personal del individuo.

Sentir una emoción es asunto sencillo. Consiste en tener imágenes mentales que surjan de patrones neuronales que representen los cambios en el cuerpo y el cerebro que conforman una emoción. Pero saber que tenemos ese sentimiento, "sentir" ese sentimiento, ocurre solo después de que construimos las presentaciones de segundo orden necesarias para la consciencia nuclear. Como ya lo planteamos, se trata de representaciones de la relación entre el organismo y el objeto (que en este caso es una emoción) y del efecto causal de este objeto en el organismo (Damasio, 2000, p. 306).

La expresión artística supone aquí un canal facilitador de la construcción y gestión del sentimiento, pero además entendemos que el valor de la expresión artística es que conglomera o cataliza esa vinculación del sentimiento con el elemento.

- Patrimonialización: que una cosa sea considerada patrimonio es el resultado del proceso comentado anteriormente. A este resultado final se le conoce como patrimonialización:

Entendemos el proceso de patrimonialización como el proceso por el cual se genera un vínculo patrimonial, en cuanto a que el individuo compone unos valores propios para el objeto al que se enfrenta, un aprendizaje significativo asociado a dicho patrimonio. Al otorgar valores, el individuo da identidad a la obra y se identifica ante ella, o con ella frente a la sociedad en un proceso en el que la obra forma ya, parte del yo (Gómez-Redondo, 2011, p. 111).

¿Por qué recurrir entonces a la experimentación sensorial y la expresión plástica como recurso y fomento del proceso de patrimonialización? El alumno parte de una 
experiencia en su entorno físico, social y cultural próximo, a través de la expresión artística para generar y gestionar sus sentimientos y sensaciones que además quedan plasmados/simbolizados en una obra con diferentes materiales, con diferentes técnicas que se conforma como su patrimonio personal y presente.

\section{Conclusiones}

Una vez demostrada y fundamentada la necesidad de aplicar en el aula el patrimonio como contenido educativo, es fundamental que el docente conozca los medios, métodos y recursos con los que puede contar para cumplir los objetivos propuestos. A la hora de diseñar una propuesta didáctica, se deben desarrollar actividades dinámicas, que vinculen el centro escolar con el resto del barrio o localidad Amaral (2013), actividades que partan siempre de diversos centros de interés del niño y que fomenten un aprendizaje significativo.

Nuestra principal aportación parte de la premisa de que las actividades deben contar con la experimentación y con la intervención sensorial para fomentar el proceso de enseñanza-aprendizaje y asentar un conocimiento creado por él mismo, que fundamentarán el proceso de patrimonialización. A este respecto las actividades sensoriales son esenciales para la generación de sentimiento/conocimiento que reafirmarán el proceso detallado anteriormente: percepción-emoción-expresión/ construcción de sentimiento-patrimonialización y que llenarán al alumno de experiencias con sus respectivos valores.

Finalmente, e intentando de nuevo contextualizar esta aportación en el aula, podemos encontrar que uno de los obstáculos a la hora de llevar al aula la educación patrimonial en la etapa de infantil la es el carácter abstracto del tema, desde su definición hasta su estructura y composición. En un intento de salvar este obstáculo hemos expuesto el diseño didáctico empleado para esta investigación (en el que se implementa la constelación propuesta), que pretende, ofrecer escenarios y situaciones diversas que generen emociones, sensaciones y sentimientos a través de la experimentación, de la manipulación y del contacto directo.

Si el docente pretende que el valor patrimonial de un niño surja de sí mismo, de su propio interés y sea un reflejo de su persona, las actividades plásticas y la expresión artística deben ser una puerta abierta a este objetivo. El niño crea una obra a partir de una experiencia vivida en su entorno y a través del proceso creativo ésta va adquiriendo sentido, apareciendo el germen de un valor patrimonial hacia el elemento. 


\section{Referencias bibliográficas}

Amaral, L. (2013). Patrimonios migrantes: geopolítica e identidades en tránsito. En R. Huerta y R. De la Calle. (Coords.). Patrimonios Migrantes (pp. 95-106). Valencia: PUV.

Bloom, B.S. (1972) (Ed.). Taxonomía de las Metas Educativas, por una comisión de Examinadores de Enseñanza Técnica y Universitaria. Tomo I: Ámbito del Conocimiento. Alcoy: Marfil.

Calaf, R. y Fontal, O. (2007). Metáforas para conceptualizar el patrimonio artístico y su enseñanza. En R. Huerta y R. De la Calle. (Coords.). Espacios estimulantes. Museos y educación artística (pp. 67-91). Valencia: PUV.

Calaf, R. (2009). Didáctica del patrimonio: epistemología, metodología y estudio de casos. Gijón: Trea.

Cardo, C., Vila, B. (2005). Material sensorial (0-3 años) Manipulación y experimentación. Barcelona: Graó.

Cuenca, J. M. (2014). El papel del patrimonio en los centros educativos: hacia la socialización patrimonial. Tejuelo: Didáctica de la Lengua y la Literatura, 19, 76-96.

Damasio, A. (1999). El error de Descartes. Santiago de Chile: Andrés Bello.

Damasio, A. (2000). Sentir lo que sucede. Cuerpo y emoción en la fábrica de la consciencia. Andrés Bello: Santiago de Chile.

Fontal, O. (2003). La educación patrimonial: teoría y práctica para el aula, el museo e internet. Gijón: Trea.

Fontal, O. (2006). Claves del patrimonio cultural del presente y desde el presente para abordar su enseñanza, Pulso, 29, 9-21.

Gómez-Redondo, C. (2011). Procesos de Patrimonialización en el Arte Contemporáneo. EARI. Educación Artística Revista de Investigación, 2, 108-112.

Gómez-Redondo, C. (2013). Procesos de patrimonialización en el arte contemporáneo. Diseño de un artefacto educativo para la identización. Tesis doctoral inédita. Universidad de Valladolid. 
GÓMEZ \& FENOY / La sensorialidad como estrategia para la educación patrimonial en el aula de educación infantil

Gómez-Redondo. C. (2014). El elemento afectivo como conglomerante en los procesos de patrimonialización. EARI. Educación Artística Revista de Investigación, 5, 56-70. doi: 10.7203/eari.5.3324

Huerta, R. y de la Calle, R. (2013). Patrimonios Migrantes. Valencia: PUV.

Huerta, R: (2013). La identidad como geografía liminar. Nuevas ideas para la educación en artes visuales. Aula de innovación educativa, 220, 12-17.

Juanola, R., Calbó, M. y Vallés, J. (2006). Educació del patrimoni: visions interdisciplinàries. Girona: Documenta Universitaria.

Spinoza, B. (1958). Ética demostrada según el orden geométrico. México D.F.: Fondo de Cultura Económica.

Vicent, N. e Ibáñez, A. (2012). El uso de las nuevas tecnologías y el patrimonio en el ámbito escolar. Aula de Innovación Educativa, 208, 22-27.

Vigotsky, L.S. (1998). La imaginación y el arte en la infancia. Madrid: Akal. 